\title{
Screw Dislocations in GaN
}

\author{
Z. Liliental-Weber, J. Jasinski, J. Washburn and M.A. O'Keefe \\ Lawrence Berkeley National Laboratory, MSD, 1 Cyclotron Road, Berkeley, CA 94720, USA
}

GaN has received much attention over the past few years because of several new a pplications, including light emitting diodes, blue laser diodes [1] and high -power microwave transistors [2]. One of the biggest problems is a high density of structural defects, mostly dislocations, due to a lack of a suitable lattice-matched substrate si nce bulk GaN is difficult to grow in large sizes. So far, the substrate of choice has been sapphire $\left(\mathrm{Al}{ }_{2} \mathrm{O}_{3}\right)$, which has a $14 \%$ lattice -size mismatch and a $34 \%$ mismatch in thermal expansion coefficient. As a result of growth along (0001) $\mathrm{GaN}$ on $\mathrm{Al}{ }_{2} \mathrm{O}_{3}$, high concentrations of misfit and threading dislocations are formed. The main concerns are threading dislocations because they will propagate to the active parts of devices grown on top of the underlying $\mathrm{GaN}$ layers, due to the fact that dislocations cannot termi nate inside the material unless they form half-loops. One of the growth techniques that give smaller dislocation density is hydride vapor-phase epitaxy (HVPE). The lower density is due to the fact that large thickness of GaN can be grown, allowing more interactions between dislocations and lowering their density [3].

Transmission Electron Microscopy (TEM) has been applied to study these layers in plan -view and cross-sections on samples prepared by conventional techniques such as mechanical thinning and precision ion milling. The density of dislocations close to the sample surface of a $1 \mu \mathrm{m}$-thick HVPE sample was in the range of $3 \times 10^{9} \mathrm{~cm}^{-2}$. All three types of dislocations were present in these samples, and almost 50\% were screw dislocations (Fig.1a). When the diffraction condition $\mathbf{g . b}=0$ was applied in cross-sectioned samples so that screw dislocations were out of contrast, small features along each screw dislocation line were still observed (Fig.1b -d). A drastic change of thickness, and a change of contrast fr om black to white on the edges of these features, was observed when conditions were changed from overfocus to underfocus. These changes allowed us to define the features as voids. In the [1120] projection, these voids had a triangular shape with a base siz e of about $3 \mathrm{~nm}$ and a height of about $4 \mathrm{~nm}$. The voids were arranged on top of each other, with the direction from the tip to the base along the $\mathrm{Ga}$ to $\mathrm{N}$ bond length parallel to the c-axis (Ga polarity). Small gaps could be observed between these voids ( $\mathrm{Fi}$ g.1b,d). In plan -view configuration the voids have hexagonal shape. Amorphous-looking features, probably due to TEM sample preparation, could be seen inside the voids when focus was set at the top of the void. By changing the defocus, one can reach the tip of the void and observe crystallographic planes around and below the void (Fig.1f).

Voids were not observed along edge dislocations in the same sample. Along some of the screw dislocations, nanotubes that are also empty inside were observed. Similar voi ds or nanotubes were observed in MOCVD materials [4] and we have suggested that the reason for their formation can be contamination - most probably by oxygen. Theoretical predictions [5] confirm our suggestion and show the possibility of $\mathrm{V}_{\mathrm{Ga}}-\left(\mathrm{O}_{\mathrm{N}}\right)_{3}$ pair for mation along particular crystallographic planes. When such complexes are formed, the GaN does not grow on these planes, leading to void formation.

Segregation of impurities along a dislocation line is a known phenomenon. The fact that these voids are forme $\mathrm{d}$ along screw but not along edge dislocations, suggests that void formation might be related to the core structure of the dislocation. For the screw dislocation, the strain field along [0001] is symmetrical in all directions. For the edge dislocation, comp ressive and tensile stress on both sides of the dislocation line can make void formation impossible.

EDX and EELS mapping studies were performed in order to confirm the presence of oxygen. The results turned out to be negative, probably due to the fact th at the concentration of oxygen is low and beyond the detectability limit of our instrument. We strongly believe that some light element contamination must lead to the formation of such voids, since heavier elements (which would be easier to detect) were $n$ ot found. We observe screw dislocations in the MBE layers, (a much cleaner growth method compared to the HVPE), grown on the top of HVPE layers. These observations confirm our assumption, and show that no more voids are formed in the MBE material even tho ugh they were formed in the HVPE layer (Fig.1e). Plan -view images of screw dislocations in MBE 
layers do not show any voids (Fig.1g). These studies might suggest that the core structure of screw dislocations in the same material might differ when the mater ial is grown by different methods. Studies are in progress to confirm this hypothesis by applying a direct reconstruction of the phase and amplitude of the scattered electron wave from a focal series of high-resolution images [6].

[1]. S. Nakamura, M. Senoh, S. Nagahama, et al Appl. Phys. Lett. 72, 211 (1998).

[2]. R.J. Trew, M.W. Shin, and V. Gatto, Solid State Electr. 41, 1561 (1997).

[3] J. Jasinski and Z. Liliental-Weber, J. Electr. Mater. (2002) in press.

[4] Z. Liliental-Weber et al., Phys. Rev. Lett. 79, 2835 (1997)

[5] J.Elsner, R.Jones et al., Appl. Phys. Lett. 73, 3530 (1998)

[6] Work supported by the Director, Office of Science, Office of Basic Energy Sciences, Material Sciences Division, of the U.S. Department of Energy, under contract No. DE-AC03-76SF00098.
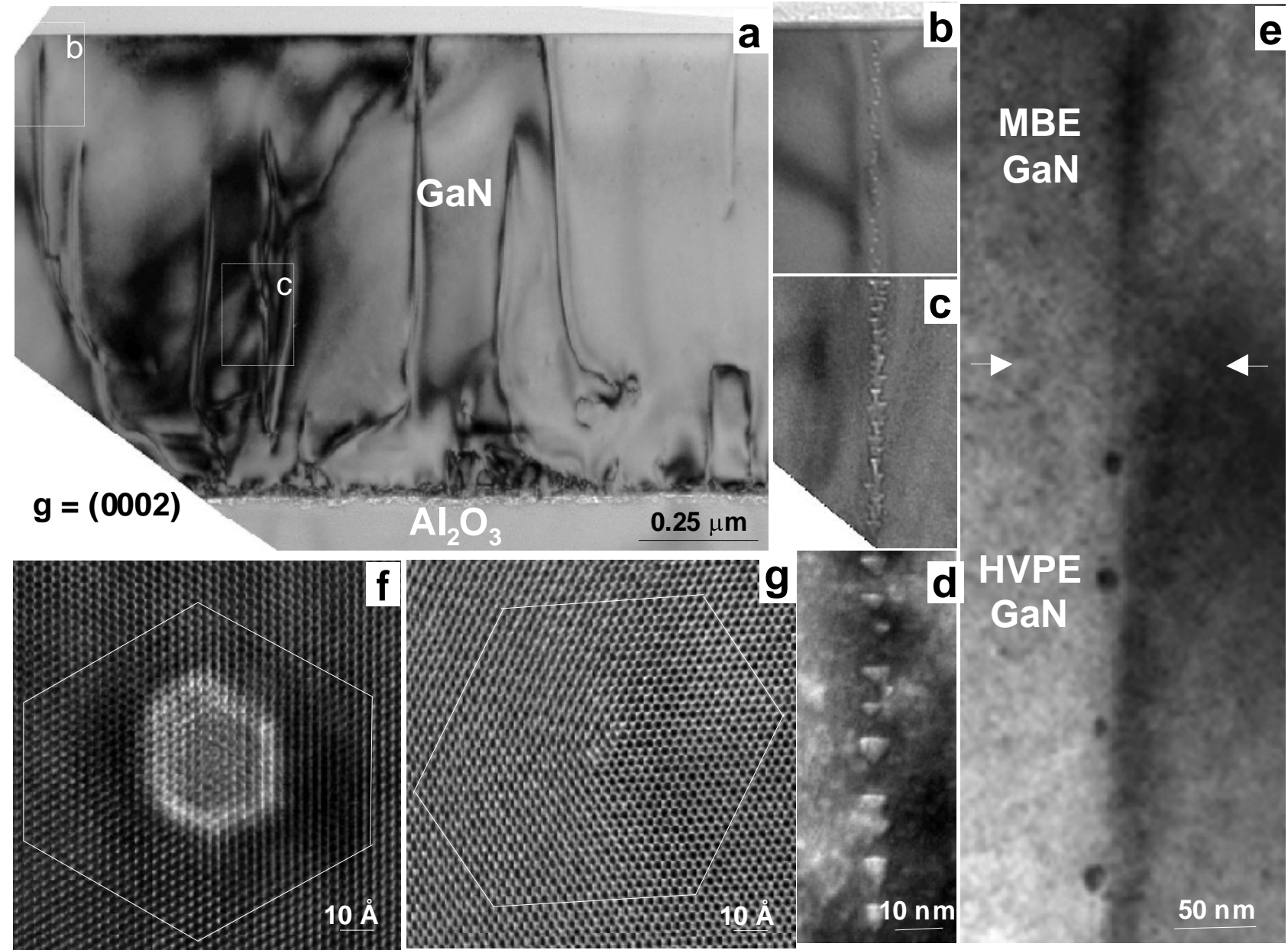

Figure 1. TEM images of screw dislocations in $\mathrm{GaN}$ grown on $\mathrm{Al}$

${ }_{2} \mathrm{O}_{3}$. (a) Cross -section view of HVPE GaN with screw dislocations visible for $\mathbf{g}=(0002) .(\mathrm{b}, \mathrm{c})$ Higher magnification images of screw dislocations out of contrast, $\quad \mathbf{g}=\left(\begin{array}{ll}11 & 20\end{array}\right)$, from areas marked on (a) - note voids along dislocation lines. (d) Voids are stacked along the Ga to $\mathrm{N}$ bond length parallel to the c-axis - note the separation between adjacent voids. (e) Screw dislocation propagates from HVP E GaN (lower) to MBE GaN (upper) through interface (arrowed), but voids formed in HVPE are not present in the MBE material. High-resolution plan views of screw dislocations show that pits form around screws in HVPE GaN (f) but not in MBE GaN (g) - white lines describe Burgers circuits around the screw dislocations . 